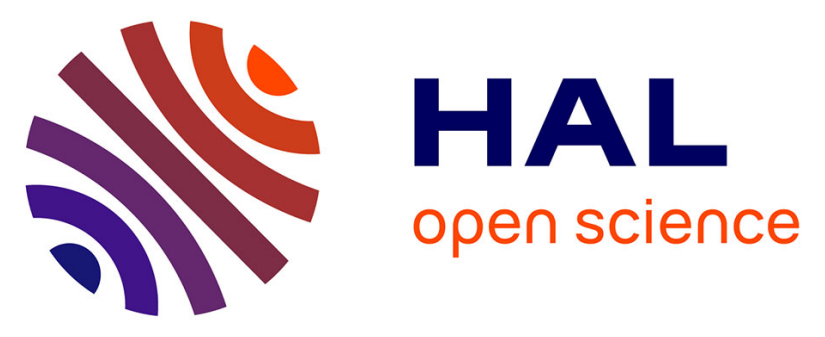

\title{
Evaluation of matrix-assisted laser desorption ionization-time of flight mass spectrometry for identification of human oral Capnocytophaga species
}

\author{
Anne Jolivet-Gougeon, Nicolas Helsens, Elise Renard, Zohreh \\ Tamanai-Shacoori, Martine Bonnaure-Mallet
}

\section{To cite this version:}

Anne Jolivet-Gougeon, Nicolas Helsens, Elise Renard, Zohreh Tamanai-Shacoori, Martine BonnaureMallet. Evaluation of matrix-assisted laser desorption ionization-time of flight mass spectrometry for identification of human oral Capnocytophaga species. Anaerobe, 2017, 48, pp.89-93. 10.1016/j.anaerobe.2017.07.003 . hal-01619482

HAL Id: hal-01619482

https://hal-univ-rennes1.archives-ouvertes.fr/hal-01619482

Submitted on 12 Dec 2017

HAL is a multi-disciplinary open access archive for the deposit and dissemination of scientific research documents, whether they are published or not. The documents may come from teaching and research institutions in France or abroad, or from public or private research centers.
L'archive ouverte pluridisciplinaire HAL, est destinée au dépôt et à la diffusion de documents scientifiques de niveau recherche, publiés ou non, émanant des établissements d'enseignement et de recherche français ou étrangers, des laboratoires publics ou privés. 
2 Evaluation of Matrix-Assisted Laser Desorption Ionization-Time Of Flight Mass

4

Anne Jolivet-Gougeon ${ }^{1,2}$, Nicolas Helsens ${ }^{1}$, Elise Renard ${ }^{1}$, Zohreh Tamanai-Shacoori ${ }^{1}$, and

6 Martine Bonnaure-Mallet ${ }^{1,2}$

$9{ }^{1}$ Université de Rennes, INSERM U 1241, INRA, Nutrition Metabolisms and Cancer, 2 avenue 10 du Professeur Léon Bernard, 35043 Rennes, France

${ }^{2}$ CHU Pontchaillou, 2 rue Henri Le Guilloux - 35033 RENNES Cedex 9, France

Anne Jolivet-Gougeon, Université de Rennes, INSERM NuMeCan/CIMIAD, 2, avenue du

23 Professeur Léon Bernard, 35043 Rennes, France

24 Phone: (33) 223234905 - Fax: (33) 223234913

25 E-mail: anne.gougeon@univ-rennes1.fr 


\section{Abstract}

Matrix-assisted laser desorption ionization-time of flight mass spectrometry (MALDITOF MS) was evaluated for rapid identification of $c f x A$ PCR positive and negative Capnocytophaga strains. Colonies were grown on blood agar, incubated anaerobically at $37^{\circ} \mathrm{C}$ for $48 \mathrm{~h}$, and were then evaluated by MALDI-TOF MS and $16 \mathrm{~S}$ rRNA gene sequencing. Both methods identified all colonies to the genus level. The MALDI-TOF MS method gave the same result, at the species level, as 16S rRNA gene sequencing for 41/53 Capnocytophaga sp. strains (77.4\%), but the limit of this technique was the absence of some species (C. leadbetteri, C. AHN) in the Biotyper-Bruker ${ }^{\circledR}$ database used in this study. Distinction between the cefotaxime resistant and susceptible strains was unsuccessful using the MALDITOF MS method. This technique had low discriminatory power to rapidly detect betalactamase-producing Capnocytophaga strains in clinical samples. However, the results from a score-oriented dendrogram confirmed MALDI-TOF MS is a rapid, inexpensive, and reliable method for Capnocytophaga species identification. Enrichment of the reference database used (Biotyper ${ }^{\circledR}$ ) will improve future results.

Key words: MALDI-TOF Mass Spectrometry; Capnocytophaga; cfxA; $16 \mathrm{~S}$ rRNA; PCR; dendrogram 


\section{Introduction}

Capnocytophaga are part of the normal bacterial flora of the oral cavity of humans and animals. Generally, they are considered commensal bacteria but, in immunocompetent or immunocompromised patients, they can be responsible for systemic diseases [1]. These bacteria belong to the family Flavobacteriaceae, order Flavobacteriales, class Flavobacteria,

Matrix-assisted laser desorption ionization-time of flight mass spectrometry (MALDI-

TOF MS) is based on the protein composition of the microbial cell, particularly ribosomal protein contents, and can discriminate clinical and environmental species. This technique is

effective to identify anaerobic bacteria in the routine clinical microbiology laboratory setting [2-4]. Some clinical case reports used this technique to correctly identify Capnocytophaga species at the genus level $[5,6]$. extended-spectrum $\beta$-lactamase (ESBL) $[7,8]$ and carbapenemases $[2,9,10]$. Only a few studies screened beta-lactamases in anaerobes, such as the class B metallo-beta-lactamase encoded by cfiA in Bacteroides fragilis [2] or carbapenemases [9, 11]. The prevalence of beta-lactamase producing Capnocytophaga sp. is increasing in the human oral cavity, as 
71 cause of third generation cephalosporin resistance in Capnocytophaga sp. The cfxA gene, conferring constant resistance to third generation cephalosporins, especially cefotaxime

73 [15], has been described on plasmids and/or on chromosomes harbouring mobile genetic elements. This ability to gene mobilisation can be responsible for resistance gene dissemination and transfer [12]. When a beta-lactamase-producing Capnocytophaga strain is detected in a clinical sample, the empirical antimicrobial treatment administered to the patient should often be rapidly modified. However, the detection of the $c f x A$ gene is not sufficient to assert the resistance. In previous work, we showed that the expression of this gene is not always constant: if 1 st and 2 nd generation cephalosporins are most often

80 affected by the CfxA beta-lactamase, the phenotypic expression of resistance for cephalosporins of 3rd generation is reported variable according to the strains [16]. Expression of $c f x A$ genes ( $c f x A$ mRNA levels) was quantified by using quantitative PCR, in cfxA PCR-positive isolates differentiated by their beta-lactam resistance profiles. One isolate remained susceptible to beta-lactams (despite a positive $c f x A P C R$ ), and the $c f x A$ gene was not expressed ( $C_{t}$ value identical to distilled water used as negative control). But the normalized $C_{t}$ values for the third-generation cephalosporins were not directly related to MIC levels. This expression could also be influenced by replacing the sequence in the $c f x A$ gene, and enhanced expression related to the proximity of mobile elements such as mobA [16]. A simple and rapid method for detecting the presence of the CfxA beta-lactamase would therefore be useful for better interpreting the PCR results. Wybo et al. [2] managed to

91 differentiate cfiA-positive from cfiA-negative isolates in a routine laboratory setting and so pinpoint $B$. fragilis strains potentially resistant to carbapenems. In the same way, we hoped 
93 to discriminate negative and positive $c f x A$ strains: culture of bacteria with cefotaxime was

94 also tested to confirm the experiment.

95 The aim of this study was to use MALDI-TOF MS for rapid species identification of 96 Capnocytophaga. The study also tested the discriminatory power of this technique to 97 differentiate the cefotaxime resistant from cefotaxime susceptible Capnocytophaga strains.

\section{Materials And Methods}

\subsection{Strains used}

101 Reference strains ( $\mathrm{n}=5$ ) were used for this study: C. sputigena ATCC 33595, C. sputigena ATCC 33612, C. gingivalis ATCC 33624, C. ochracea ATCC 27872, and C. granulosa ATCC 51502. Clinical strains of Capnocytophaga sp. $(n=48)$ were isolated from routine cultures, at

104 the Teaching Hospital of Rennes (France). Clinical strains were isolated from oral swabs $105(n=30)$ and sputum samples $(n=8)$ on TBBP (Trypticase-blood-bacitracin-polymyxin B) agar 106 [17], and from surgical bone samples $(n=4)$, abscesses $(n=4)$, and blood samples $(n=2)$ on 107 horse blood agar (Oxoid). All samples were conserved in frozen stock cultures (Cryobanks, 108 MAST diagnostics), and cultured on horse blood agar in an anaerobic chamber for 24 to $48 \mathrm{~h}$ at $37^{\circ} \mathrm{C}$ for further experiments.

$111 \quad 2.2$ Susceptibility testing

112 The MICs were determined by Etest methodology (Etest ${ }^{\circledR}$, BioMérieux, France), and

113 interpreted according to CLSI recommendations for anaerobes [18], since no breakpoint for 114 third generation cephalosporins (as cefotaxime) is currently available for anaerobes, 115 according to EUCAST recommendations [19]. The CLSI breakpoints of cefotaxime for 
116 susceptible, intermediate, and resistant strains were $\leq 16 \mathrm{mg} / \mathrm{L}, 32 \mathrm{mg} / \mathrm{L}$, and $\geq 64 \mathrm{mg} / \mathrm{L}$,

117 respectively.

\subsection{PCR amplification}

120 Amplification of the $c f x A$ gene by PCR was performed as previously described [20]. Briefly,

121 the primers used were: cfxA1 (5'-CTTTGTCGGCAAATAAAGAT-3') and cfxA4 (5'-

122 TGAACGAGGAATGAGTGTGG-3'), generating a $580 \mathrm{bp}$ fragment and cfxA5 (5'-

123 TGGTTAATGTCGCTCAAACA-3') and cfxA6 (5'-TCAAAGCAAGTGCAGTTTAAGA-3'),

124 generating a 507bp fragment. A 966-bp consensus sequence of $c f x A 3$ was then determined.

125 The amplification conditions were $94^{\circ} \mathrm{C}$ for $1 \mathrm{~min}, 55^{\circ} \mathrm{C}$ for $1 \mathrm{~min}$, and $72^{\circ} \mathrm{C}$ for $1 \mathrm{~min}(35$

126 cycles).

127 Sequencing of the 16S rRNA gene was used as a reference method for Capnocytophaga

128 species identification, based on previous work [21]. Real-time PCR was performed with Sybr

129 green to target the $5^{\prime}$ part of the $16 \mathrm{~S}$ rRNA gene (forward primer $27 \mathrm{~F}, 5^{\prime}$-AGA GTT TGA TCM

130 TGG CTC AG-3'; reverse primer 685R3, 5'-TCT RCG CAT TYC ACC GCT AC-3'; 658-bp

131 amplification product; GenBank accession number NR 024570). The corresponding

132 amplicons were sequenced in both strands and assembled, and the consensus sequences

133 were compared with those in the Bioinformatics Bacteria Identification (BIBI) and BLAST

134 databases. The rates of concordance between 16S rRNA gene PCR and bacteriological results

135 were based on results at the genus ( $\geq 96 \%$ similarity) and species ( $\geq 98 \%$ similarity) levels.

136 Positive and negative controls were added in each series [21]. To control DNA extraction and

137 confirm the absence of PCR inhibitors, a fragment of the human beta-globin gene was

138 amplified for each negative sample. 

MS)

MALDI-TOF MS (Biotyper, Bruker $\left.{ }^{\circledR}\right)$ analyses were performed in positive linear mode in the range of $2000-20,000$ masses-to-charge ratio $(\mathrm{m} / \mathrm{z})$, and used to identify the isolates to the

144 level of species. Colonies were selected after culturing on horse blood agar in an anaerobic

145 chamber for 24 to $48 \mathrm{~h}$, in the absence of antibiotic or around to the cefotaxime disk

146 (BioRad, $30 \mu \mathrm{g}$ ). Colony spotting was performed in triplicate and spectra were obtained and

147 analyzed with MALDI Biotyper 2.0 software and references (Bruker ${ }^{\circledR}$ Daltonik, GmbH,

148 Bremen, Germany), according to the manufacturer's instructions. For the direct colony

149 method, bacteria were applied as a thin film onto a 96-spot steel plate (Bruker ${ }^{\circledR}$ Daltonics)

150 and allowed to dry at room temperature. Subsequently, $1.2 \mu \mathrm{l}$ of MALDI matrix (a saturated

151 solution of $\alpha$-cyano-4-hydroxycinnamic acid [HCCA; Bruker ${ }^{\circledR}$ Daltonics] in $50 \%$ acetonitrile

152 and $2.5 \%$ trifluoroacetic acid) was applied to the colony and allowed to dry before testing.

153 For the extraction method, 1 to 2 colonies were extracted as already described [22]. Each

154 series of measurements was preceded by calibration with a bacterial test standard (BTS

155 255343; Bruker Daltonik), to calibrate the instrument and validate the run. Negative controls

156 were a matrix deposit alone and Brucella broth (supplemented with $5 \%$ blood, vitamin $\mathrm{K} 1$

157 and cefotaxime $(0.5 \mathrm{mg} / \mathrm{L}))$. For identification, scores and reproducibility were considered

158 according to the manufacturer's instructions (Bruker ${ }^{\circledR}$ ). Results of the pattern matching

159 process were expressed as log (score) values ranging from 0 to 3 . Values from 0-1.6999

160 indicated not suitable identification, $>1.7$ indicated relationships on the genus level, and $>2.0$

161 indicated relationships on the species level (score: 2.3 to 3: high probability of identification 
162 to the species level, 2 to 2.2999: high probability of identification to the genus level and

163 probable identification to the species level, 1.7-1.999: probable identification to the genus

164 level). The interpretation considered two independent parameters: the value of the

165 homology score and the reproducibility of the identification obtained (from 10

166 measurements carried out after laser impacts where the same bacterial species must be

167 found at least three times with the highest scores).

168 To test if MALDI-TOF MS was able to differentiate between $c f x A$-negative and $c f x A$-positive

169 isolates, bacterial pellets were also extracted after incubation of each isolate in the presence

170 of cefotaxime $(0.5 \mathrm{mg} / \mathrm{L})$ in Brucella broth supplemented with $5 \%$ blood and vitamin $\mathrm{K} 1$ (1

$171 \mathrm{mg} / \mathrm{L}$ ) under anaerobic conditions for $6 \mathrm{~h}$. Identification was performed, as indicated above,

172 from a deposit of a drop of pellet left to dry and score-oriented dendrogram of matrix-

173 assisted laser desorption ionization time-of-flight mass spectrometry profiles were

174 compared.

175 The MALDI-TOF MS spectral data resulting from the analysis of 53 isolates were processed

176 using the Bruker database software to identify species-specific masses that were then used

177 to generate dendrograms. A class dendrogram of all the study isolates was constructed (with

178 the correlation distance measured by the average linkage algorithm; Euclidean distances) of

179 the Biotyper 2.0 software (Bruker Daltonics) [23]. The MALDI-TOF spectra similarity

180 dendrogram between $c f x A$ positive and negative strains, based on the mass spectral

181 patterns, was used to study dispersion of spectra [24].

182

183 3. Results

1843.1 Identifications of strains by MALDI-TOF MS and 16S rRNA gene sequencing 
isolates clearly identified all strains as Capnocytophaga (100\% agreement) at the genus level,

187 and $41 / 53(77.4 \%)$ were identified as the same at the species level (Table 1$)$, with a score

$188>1.8$ and a reproducibility $>4$ (minimum of 3/10 replicates had MALDI confidence scores

$189>1.8)$. Both methods identified the $C$. sputigena $(n=31)$ and $C$. ochracea $(n=3)$ strains. The

190 four Capnocytophaga AHN (AHN9576/AHN9798/AHN8471/ChDc) strains identified by $16 \mathrm{~S}$

191 rRNA sequencing were characterized as C. sputigena by MALDI-TOF MS. The six $C$.

192 leadbetteri strains recognized by $16 \mathrm{~S}$ rRNA sequencing were identified as Capnocytophaga.

193 sp. by MALDI-TOF MS. One strain classified as Capnocytophaga geno sp., C. granulosa and

194 another as $C$. gingivalis by $16 \mathrm{~S}$ rRNA sequencing were identified as $C$. sputigena, $C$. gingivalis

195 and C. granulosa, respectively, by MALDI-TOF MS. The MALDI-TOF MS technique had limited

196 ability to discriminate between C. gingivalis and C. granulosa (two misidentified strains), but

197 these species were similar, as shown on the dendrogram of all Capnocytophaga species

198 identified using MALDI-TOF MS (Figure 1).

\section{$199 \quad 3.2 \quad$ Susceptibility testing}

200 From the total clinical Capnocytophaga sp. ( $n=48), 35$ strains were cefotaxime-susceptible, 201 three were intermediate, and 10 were resistant, according to CLSI criteria [17]. The MICs of 202 cefotaxime were: $\mathrm{MIC}_{50}=1.5$ and $\mathrm{MIC}_{90} \geq 256 \mathrm{mg} / \mathrm{L}$ (range: $0.016->256 \mathrm{mg} / \mathrm{L}$ ). Among these 203 Capnocytophaga sp. isolates, the $c f x A$ gene was amplified by PCR in 42 (87.5\%) all resistant 204 to cefalotin (data not shown): 13 strains were resistant/intermediate and 29 remained 205 phenotypically susceptible to cefotaxime despite a positive $c f x A$ PCR. Five of the latter (four 206 from saliva and one from osteo-articular fluid) $c f x A+$, susceptible to cefotaxime with MIC 207 range: $0.016-0.5 \mathrm{mg} / \mathrm{L}$ were found to be gene misexpressed and/or beta-lactamase 
208 inactivated, as previously suggested. The five reference strains were $c f x A$ PCR negative and

209 susceptible to beta-lactams.

2103.3 MALDI-TOF spectra similarity dendrogram

211 The MALDI-TOF spectra similarity dendrogram, in which the strains were dispersed

212 irrespective of their mass spectral patterns, was unsuccessful in differentiating susceptible 213 and resistant strains (data not shown).

4. Discussion

In a previous study, Conrads et al. [25] used oligonucleotide probes and

217 demonstrated that the dendrogram of $16 \mathrm{~S}$ rRNA sequences of the Capnocytophaga

218 reference strains showed a very close relationship, especially C. granulosa and C. gingivalis

219 (97.7\% similarity) and C. ochracea and C. sputigena (95.0\% similarity). Previously, MALDI-TOF

220 MS was used with success to identify strict anaerobes, such as Bacteroides [26] or Prevotella

221 [27], with clinical isolates of Prevotella sp. identified to the species (62.7\%) and genus

222 (73.5\%) levels. Recently, Magnette et al. [28] showed that MALDI-TOF MS provided a reliable

223 identification to the species level for $100 \%$ of 45 blind-coded Capnocytophaga canimorsus

224 and C. cynodegmi, but only following the establishment of a complementary homemade

225 reference database. Using MALDI-TOF MS in this study, we found improved Capnocytophaga

226 genus level identification (100\%) and a good human oral Capnocytophaga species level

227 identification (77.4\%). The six C. leadbetteri and four C. AHN strains (recognized by $16 \mathrm{~S}$ rRNA

228 sequencing) were identified by MALDI-TOF MS as C. spp. and C. sputigena, respectively. This

229 result was not surprising because $C$. leadbetteri and $C$. AHN do not exist in the Biotyper-

230 Bruker ${ }^{\circledR}$ database used, as was previously observed [4]. In addition, the MALDI-TOF MS 
method could not completely discriminate between C. gingivalis and C. granulosa, a finding

232 that is consistent with previous reports using multilocus enzyme electrophoretic analysis and

233 serotyping of immunoglobulin A1 proteases [29]. Thus, our results suggest that MALDI-TOF

234 MS can be used for rapid and reliable identification of most clinical Capnocytophaga strains.

We also investigated if MALDI-TOF MS could be used to detect the presence of the

236 beta-lactamase CfxA (mainly responsible for cefotaxime resistance) or one of its metabolites

237 in Capnocytophaga strains. The MALDI-TOF MS method failed to detect cfxA positive

238 Capnocytophaga strains (compared to $c f x A$ negative strains), cultured in the presence or

239 absence of cefotaxime. Most previous studies used inhibitors of beta-lactamases (ESBL or

240 carbapenemases) to reveal by MALDI-TOF MS the intermediates of beta-lactamases [30] or

241 enzyme inhibition [31]. We did not do this because the intrinsic activity of all beta-lactamase

242 inhibitors was demonstrated in Capnocytophaga species [32]. Wybo et al. [2] demonstrated

243 that MALDI-TOF MS could be used to differentiate between cfiA-positive and cfiA-negative

244 Bacteroides strains, cultured on fastidious anaerobic agar at $35^{\circ} \mathrm{C}$ in an anaerobic chamber

245 for 24 to $48 \mathrm{~h}$, without use of antibiotic or beta-lactamase inhibitors. This result was

246 confirmed by Fenyvesi et al. [33], who characterized 5/60 cfiA positive strains using this

247 method. In our study, we could not discriminate between cefotaxime susceptible (cfxA-

248 positive) and resistant ( $c f x A$-negative) strains using the MALDI-TOF MS method and MALDI

249 Biotyper 2.0 software, as recommended by Wybo et al. [2].

250 The limits of this technique for beta-lactamase detection have already been

251 highlighted. Trevino et al. [11] used MALDI-TOF MS for rapid characterization of

252 carbapenemase-producing Enterobacteriaceae and genetic diversity among carbapenemase-

253 producing strains. Trevino et al. [11] also showed that fingerprinting analyses by automated 
254 repetitive extragenic palindromic-polymerase chain reaction (rep-PCR) (DiversiLab system)

255 showed higher discriminatory power than MALDI-TOF MS for clonal strain typing. With this

256 last method, distinct mass spectra were obtained only within a mass range of 2,000 to

257 20,000 Da using whole cell MALDI-TOF MS [34], and the mass range of the CfXA beta-

258 lactamase was estimated to 35,275 Da and CfiA to be 27,260 Da. By MALDI-TOF MS, only

259 degradation products of the beta-lactam cefotaxime might have been detected, but no

260 difference in profiles was observed when Capnocytophaga strains were cultured with and

261 without antibiotic. Moreover, the expression of CfxA enzyme has been described as variable

262 with different MICs reported. Wybo et al. [2] suggested that the cfiA gene was not always

263 activated, and Soki et al. [35] hypothesized that the cfiA gene was activated by its own

264 promotor. The differentiation of cfiA-negative and cfiA-positive Bacteroides fragilis [2] was

265 probably due to a selection bias in identifying strains, because the cfiA gene, encoding the

266 unique carbapenemase found in Bacteroides, was restricted to division II Bacteroides fragilis

267 strains [36].

\section{Conclusion}

In conclusion, MALDI-TOF MS can be used for rapid and low cost oral human

271 Capnocytophaga genus identification. But the limits of this technique was the absence of

272 some species (C. leadbetteri, C. AHN) in the Biotyper-Bruker ${ }^{\circledR}$ database used in this study,

273 and its inability to completely discriminate between C. gingivalis and C. granulosa. This 274 method was not also suitable for quickly detecting beta-lactamase producing 275 Capnocytophaga strains. 
We thank Philippe Gautier for his help with sequence analysis, Nolwenn Oliviero for

technical assistance, Adina Pascu for help in formatting the manuscript, and San Francisco

Edit for help in translating the manuscript.

Compliance with ethical standards: no funding to declare; the authors declare no conflict of

interests; ethical approval: not applicable; Informed consent: not necessary.

\section{References}

[1] J.D. Beck, P. Eke, G. Heiss, P. Madianos, D. Couper, D. Lin, et al., Periodontal disease and coronary heart disease: a reappraisal of the exposure, Circulation 112 (2005) 19-24.

[2] I. Wybo, A. De Bel, O. Soetens, F. Echahidi, K. Vandoorslaer, M. Van Cauwenbergh, et al., Differentiation of cfiA-negative and cfiA-positive Bacteroides fragilis isolates by matrixassisted laser desorption ionization-time of flight mass spectrometry, J. Clin. Microbiol. 49 (2011) 1961-4.

[3] O. Garner, A. Mochon, J. Branda, C.A. Burnham, M. Bythrow, M. Ferraro, et al., Multicentre evaluation of mass spectrometric identification of anaerobic bacteria using the VITEK(R) MS system, Clin. Microbiol. Infect. 20 (2014) 335-9.

[4] B. Rodriguez-Sanchez, L. Alcala, M. Marin, A. Ruiz, E. Alonso, E. Bouza, Evaluation of MALDI-TOF MS (Matrix-Assisted Laser Desorption-Ionization Time-of-Flight Mass Spectrometry) for routine identification of anaerobic bacteria, Anaerobe 42 (2016) 101-7.

[5] S. Zangenah, V. Ozenci, S. Borang, P. Bergman, Identification of blood and wound isolates of $C$. canimorsus and C. cynodegmi using VITEK2 and MALDI-TOF, Eur. J. Clin. Microbiol. Infect. Dis. 31 (2012) 2631-7.

[6] M.T. Bastida, M. Valverde, A. Smithson, F. Marco, [Fulminant sepsis due to Capnocytophaga canimorsus: Diagnosis by matrix-assisted laser desorption/ionization-time of flight mass spectrometry], Med. Clin. (Barc.) 142 (2014) 230-1.

[7] L.N. Ikryannikova, E.A. Shitikov, D.G. Zhivankova, E.N. Il'ina, M.V. Edelstein, V.M. Govorun, A MALDI TOF MS-based minisequencing method for rapid detection of TEM-type extended-spectrum beta-lactamases in clinical strains of Enterobacteriaceae, J. Microbiol. Methods 75 (2008) 385-91.

[8] R. Schaumann, N. Knoop, G.H. Genzel, K. Losensky, C. Rosenkranz, C.S. Stingu, et al., A step towards the discrimination of beta-lactamase-producing clinical isolates of Enterobacteriaceae and Pseudomonas aeruginosa by MALDI-TOF mass spectrometry, Med. Sci. Monit. 18 (2012) MT71-7.

[9] A. Johansson, E. Nagy, J. Soki, ESGAI, Detection of carbapenemase activities of Bacteroides fragilis strains with matrix-assisted laser desorption ionization--time of flight mass spectrometry (MALDI-TOF MS), Anaerobe 26 (2014) 49-52. 
[10] M. Berrazeg, S.M. Diene, M. Drissi, M. Kempf, H. Richet, L. Landraud, et al., Biotyping of multidrug-resistant Klebsiella pneumoniae clinical isolates from France and Algeria using MALDI-TOF MS, PLoS One 8 (2013) e61428.

[11] M. Trevino, P. Areses, M.D. Penalver, S. Cortizo, F. Pardo, M.L. del Molino, et al., Susceptibility trends of Bacteroides fragilis group and characterisation of carbapenemaseproducing strains by automated REP-PCR and MALDI TOF, Anaerobe 18 (2012) 37-43.

[12] T. Handal, C. Giraud-Morin, D.A. Caugant, I. Madinier, I. Olsen, T. Fosse, Chromosome- and plasmid-encoded beta-lactamases in Capnocytophaga spp, Antimicrob. Agents Chemother. 49 (2005) 3940-3.

[13] A. Jolivet-Gougeon, Z. Tamanai-Shacoori, L. Desbordes, V. Gandemer, J.L. Sixou, N. Morvan-Graveline, et al., Prevalence of oropharyngeal beta-lactamase-producing Capnocytophaga spp. in pediatric oncology patients over a ten-year period, BMC Infect. Dis. 5 (2005) 32.

[14] E. Ehrmann, T. Handal, Z. Tamanai-Shacoori, M. Bonnaure-Mallet, T. Fosse, High prevalence of beta-lactam and macrolide resistance genes in human oral Capnocytophaga species, J. Antimicrob. Chemother. 69 (2014) 381-4. et al., Genetic determinants associated with $c f x A$-positive clinical Capnocytophaga isolates, Int J Antimicrob Agents 46 (2015) 356-8.

[16] Z. Tamanai-Shacoori, C. Monfort, N. Oliviero, P. Gautier, M. Bonnaure-Mallet, A. Jolivet-Gougeon, cfxA expression in oral clinical Capnocytophaga isolates, Anaerobe 35 (2015) 68-71.

[17] E. Ehrmann, A. Jolivet-Gougeon, M. Bonnaure-Mallet, T. Fosse, Antibiotic content of selective culture media for isolation of Capnocytophaga species from oral polymicrobial samples, Lett. Appl. Microbiol. 57 (2013) 303-9.

[18] CLSI M100 S26:2016. Performance Standards for Antimicrobial Susceptibility Testing; 26th Edition p115. http://clsi.org/m100/p115.

\section{[19] EUCAST, http://www.eucast.org/clinical breakpoints/.}

[20] A. Jolivet-Gougeon, Z. Tamanai-Shacoori, L. Desbordes, N. Burggraeve, M. Cormier, M. Bonnaure-Mallet, Genetic analysis of an ambler class A extended-spectrum betalactamase from Capnocytophaga ochracea, J. Clin. Microbiol. 42 (2004) 888-90.

[21] P. Bemer, C. Plouzeau, D. Tande, J. Leger, B. Giraudeau, A.S. Valentin, et al., Evaluation of $16 \mathrm{~S}$ rRNA gene PCR sensitivity and specificity for diagnosis of prosthetic joint infection: a prospective multicenter cross-sectional study, J. Clin. Microbiol. 52 (2014) 35839.

[22] A.A. Alatoom, S.A. Cunningham, S.M. Ihde, J. Mandrekar, R. Patel, Comparison of direct colony method versus extraction method for identification of gram-positive cocci by use of Bruker Biotyper matrix-assisted laser desorption ionization-time of flight mass spectrometry, J. Clin. Microbiol. 49 (2011) 2868-73.

[23] E. Kim, Y. Cho, Y. Lee, S.K. Han, C.G. Kim, D.W. Choo, et al., A proteomic approach for rapid identification of Weissella species isolated from Korean fermented foods on MALDITOF MS supplemented with an in-house database, Int. J. Food Microbiol. 243 (2017) 9-15.

[24] A. Jung, M. Metzner, M. Ryll, Comparison of pathogenic and non-pathogenic Enterococcus cecorum strains from different animal species, BMC Microbiol. 17 (2017) 33.

[25] G. Conrads, R. Mutters, I. Seyfarth, K. Pelz, DNA-probes for the differentiation of Capnocytophaga species, Mol. Cell. Probes 11 (1997) 323-8. 
[26] E. Nagy, T. Maier, E. Urban, G. Terhes, M. Kostrzewa, E.S.G.o.A.R.i.A. Bacteria, Species identification of clinical isolates of Bacteroides by matrix-assisted laser-desorption/ionization time-of-flight mass spectrometry, Clin. Microbiol. Infect. 15 (2009) 796-802.

[27] I. Wybo, O. Soetens, A. De Bel, F. Echahidi, E. Vancutsem, K. Vandoorslaer, et al., Species identification of clinical Prevotella isolates by matrix-assisted laser desorption ionization-time of flight mass spectrometry, J. Clin. Microbiol. 50 (2012) 1415-8.

[28] A. Magnette, T.D. Huang, F. Renzi, P. Bogaerts, G.R. Cornelis, Y. Glupczynski, Improvement of identification of Capnocytophaga canimorsus by matrix-assisted laser desorption ionization-time of flight mass spectrometry using enriched database, Diagn. Microbiol. Infect. Dis. 84 (2016) 12-5.

[29] E.V. Frandsen, W.G. Wade, Differentiation of human Capnocytophaga species by multilocus enzyme electrophoretic analysis and serotyping of immunoglobulin A1 proteases, Microbiology 142 ( Pt 2) (1996) 441-8. [30] C.R. Bethel, M. Taracila, T. Shyr, J.M. Thomson, A.M. Distler, K.M. Hujer, et al., Exploring the inhibition of CTX-M-9 by beta-lactamase inhibitors and carbapenems, Antimicrob. Agents Chemother. 55 (2011) 3465-75. [31] J.E. Hugonnet, J.S. Blanchard, Irreversible inhibition of the Mycobacterium tuberculosis beta-lactamase by clavulanate, Biochemistry 46 (2007) 11998-2004.

[32] A. Jolivet-Gougeon, A. Buffet, C. Dupuy, J.L. Sixou, M. Bonnaure-Mallet, S. David, et al., In vitro susceptibilities of Capnocytophaga isolates to beta-lactam antibiotics and betalactamase inhibitors, Antimicrob. Agents Chemother. 44 (2000) 3186-8. MALDI-TOF/MS for routine detection of cfiA gene-positive Bacteroides fragilis strains, Int. J.

383 Antimicrob. Agents 44 (2014) 474-5.

384 [34] S.Y. Hsieh, C.L. Tseng, Y.S. Lee, A.J. Kuo, C.F. Sun, Y.H. Lin, et al., Highly efficient 385 classification and identification of human pathogenic bacteria by MALDI-TOF MS, Mol. Cell. Proteomics 7 (2008) 448-56.

387 [35] J. Soki, R. Edwards, M. Hedberg, H. Fang, E. Nagy, C.E. Nord, et al., Examination of cfiA-mediated carbapenem resistance in Bacteroides fragilis strains from a European antibiotic susceptibility survey, Int. J. Antimicrob. Agents 28 (2006) 497-502. negative) and division II (cfiA-positive) Bacteroides fragilis strains by matrix-assisted laser desorption/ionization time-of-flight mass spectrometry, J. Med. Microbiol. 60 (2011) 1584- 
FIGURE LEGEND

397

398 Figure 1 Score-oriented dendrogram of matrix-assisted laser desorption ionization time-of-

399 flight mass spectrometry profiles generated by the default setting in MALDI Biotyper 400 software version 2.0. for 53 Capnocytophaga strains cultured on blood agar for $48 \mathrm{~h}$ under

401 anaerobic conditions. Reference strains were C. sputigena ATCC 33595 (strain 40), C. 402 sputigena ATCC 33612 (strain 41), C. ochracea ATCC 27872 (strain 42), C. gingivalis ATCC 40333624 (strain 43), and C. granulosa ATCC 51502 (strain 44). The misidentified (compared 404 with the 16S rRNA gene sequencing identification technique) species were: . granulosa/C. 405 gingivalis $(\mathrm{n}=1)$, C. spp./C. leadbetteri $(\mathrm{n}=6)$, C. sputigena/C. AHN $(\mathrm{n}=4)$, and C. sputigena/C 406 sp. $(\mathrm{n}=1)$. Two distinct clades appeared on dendrogram using MALDI-TOF corresponding to: 407 clade at the top: clade for C. gingivalis/granulosa, and clade down: clade for other 408 Capnocytophaga spp.

409

410 
412 Table 1 The number of isolates identified at the indicated level by matrix-assisted laser

413 desorption ionization-time of flight mass spectrometry (MALDI-TOF MS) compared with 16S

414 rRNA gene sequencing results for 53 Capnocytophaga strains.

415

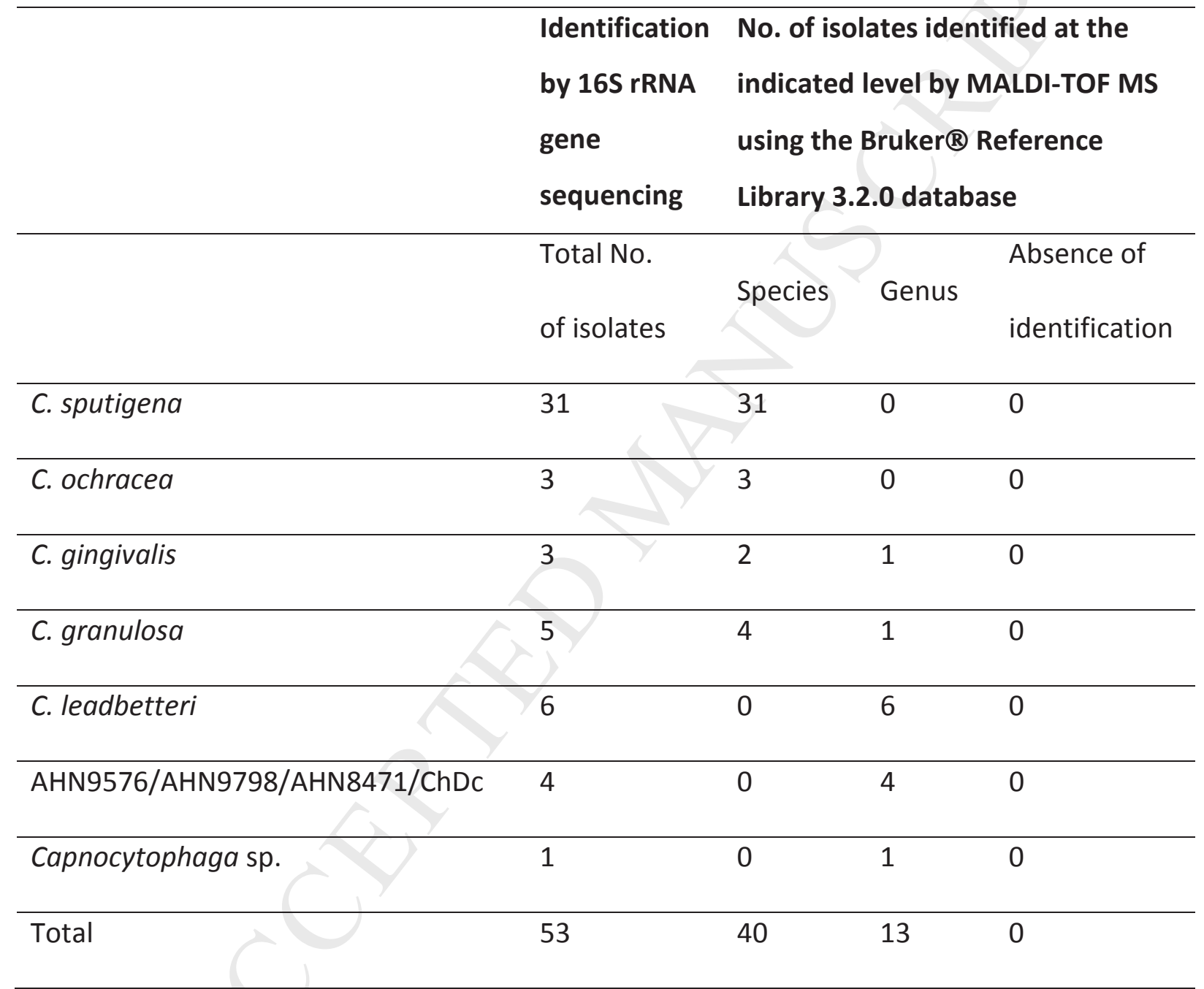

416

417 
Maldi-Tof MS

identification

MSP Dendrogram

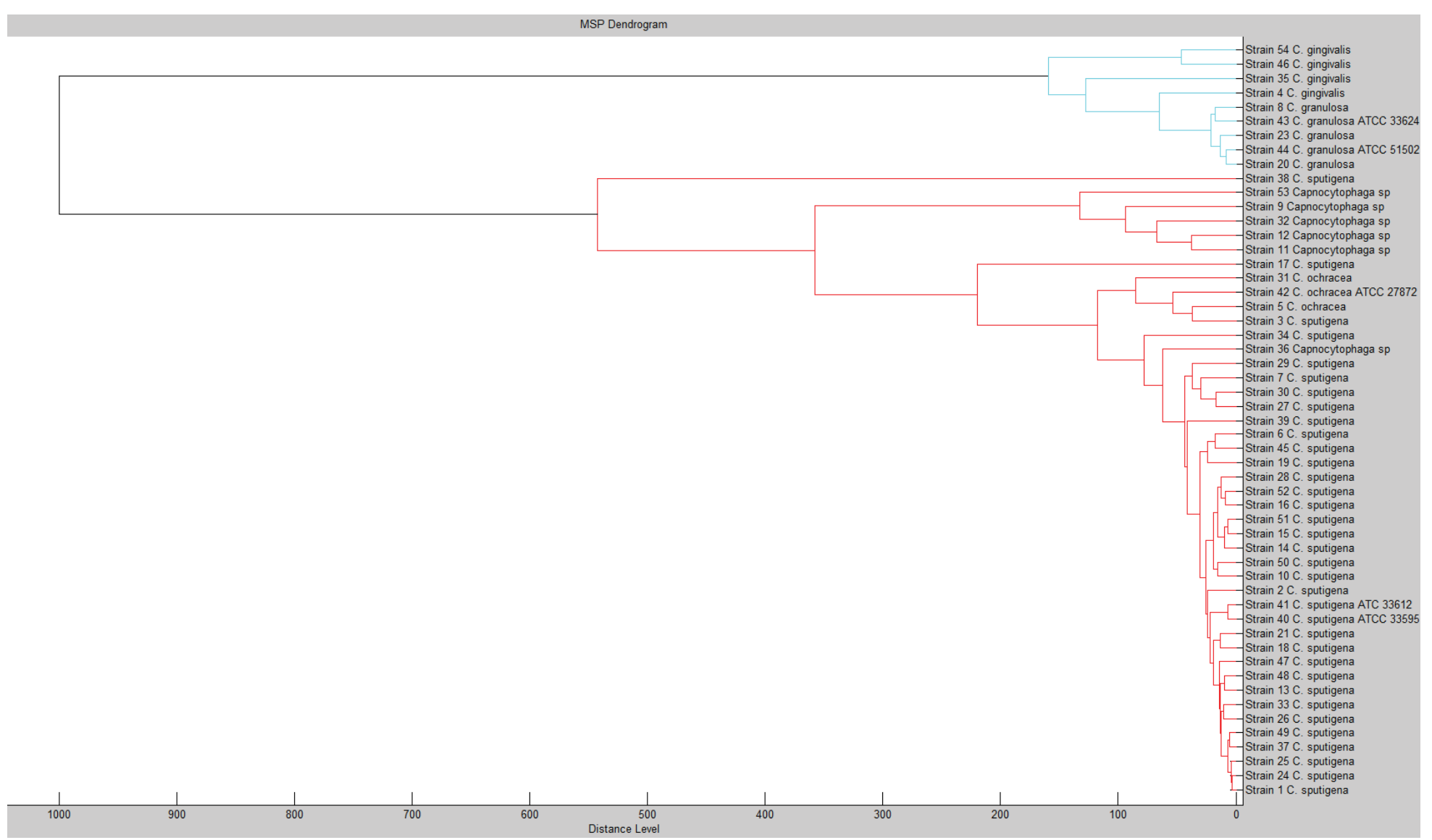

16SrRNA sequencing

identification

(strain number)

C. gingivalis (43)

C. leadbetteri (53)
c. leadbetteri (9)

c. leadbetteri(i32)

c. leachetereri(12)
c. leadbetteri(11)

HN847//ChDC (17)

c. leadbetteri (36)

AHN9576/AHN9798/AHN8471/ChDC(7)

AHN9607/AHN9576/AHN9798/AHN8471/ChDCos43 (30)

Capnocytophaga geno sp. 99\% (6)

C. AHN9576/AHN9798/AHN8471/ChDC (13) 